\title{
La loi du 11 octobre 2010 au prisme du Conseil d'État et du Conseil constitutionnel
}

Anne Levade

\section{OpenEdition}

1 Journals

Édition électronique

URL : http://journals.openedition.org/rdr/902

DOI : $10.4000 /$ rdr.902

ISSN : 2534-7462

Éditeur

Presses universitaires de Strasbourg

\section{Édition imprimée}

Date de publication : 8 novembre 2016

Pagination : 29-45

ISBN : 978-2-86820-959-7

ISSN : 2493-8637

Référence électronique

Anne Levade, «La loi du 11 octobre 2010 au prisme du Conseil d'État et du Conseil constitutionnel », Revue du droit des religions [En ligne], 2 | 2016, mis en ligne le 11 février 2020, consulté le 19 novembre 2020. URL : http://journals.openedition.org/rdr/902 ; DOI : https://doi.org/10.4000/rdr.902

\section{(c) (†) (8)}

La revue du droit des religions est mise à disposition selon les termes de la Creative Commons Attribution - Pas d'Utilisation Commerciale 4.0 International - CC BY-NC 4.0. 


\section{LA LOI DU 11 OCTOBRE 2010 AU PRISME DU CONSELL D'ÉTAT ET DU CONSELL CONSTITUTIONNEL}

\section{Anne LEVADE}

Université Paris-Est Créteil Val de Marne, Sources du droit, Institutions, Europe (SDIE)

\section{RÉSUMÉ}

De la lecture combinée de l'étude du Conseil d'État et de la décision du Conseil constitutionnel relatives à la loi du 11 octobre 2010 interdisant la dissimulation du visage dans l'espace public ressortent deux idées partagées : l'inadéquation à l'objectif poursuivi des fondements juridiques traditionnels et donc la nécessité d'un fondement novateur. Pour l'une et l'autre institution, une interdiction générale doit demeurer l'exception, justifiant que son fondement fasse l'objet d'une appréciation qui sorte elle-même de l'ordinaire. Le juge constitutionnel a ainsi dégagé une norme de référence jusque-là inexploitée.

\section{ABSTRACt}

Two main ideas are shared by the Council of State and the Constitutional Council as regards the Act of 11 October 2010 prohibiting the concealment of the face in the public space: traditional legal basis are inappropriate to achieve the objective which requires an innovative basis. For each institution, a general prohibition must remain the exception, justifying that its basis is something unique to stand out. Thus, the constitutional court has highlighted a hitherto untapped reference standard. 
l'aune de ses aspects juridiques, analyser la loi du 11 octobre 2010
interdisant la dissimulation du visage dans l'espace public au prisme du Conseil d'État et du Conseil constitutionnel peut sembler relever de l'évidence. L'un et l'autre se sont effectivement prononcés sur ce texte et l'on se souvient combien l'avis du premier et la décision du second ont, à six mois d'intervalle, alimenté le débat et suscité la polémique. Pourtant, et ici réside la difficulté, hors le fait que l'un et l'autre se sont prononcés « en droit », envisager leurs positions comme constitutives d'un prisme unique serait erroné. Plusieurs raisons permettent de l'expliquer qui supposent, en préalable, un bref rappel d'ordre chronologique.

D'abord, les dates du rapport et de la décision :

- le 30 mars 2010, le Conseil d'État rendait public un rapport, intitulé Étude relative aux possibilités juridiques d'interdiction du port du voile intégral, qui avait été adopté en assemblée générale plénière cinq jours auparavant ${ }^{1}$;

- le 7 octobre 2010, le Conseil constitutionnel publiait sa décision $\mathrm{n}^{\circ}$ 2010-613 DC, relative à la loi interdisant la dissimulation du visage dans l'espace public ${ }^{2}$.

Ensuite, entre ces deux dates, une $l o i^{3}$, donc, dont le projet a été délibéré en Conseil des ministres le 19 mai 2010, puis adopté au terme d'une unique lecture par chaque assemblée, le 13 juillet à l'Assemblée nationale et le 14 septembre au Sénat. La rapidité du processus peut surprendre si l'on songe à la vivacité du débat et alors même qu'il n'avait pas été décidé de recourir à la procédure accélérée. C'est assurément parce que si débat - et même combat - il y eut, il fut largement mené en amont et, pour partie, avant même que le Conseil d'État fût sollicité.

Car, enfin, rappelons-le, c'est, en premier lieu, le président de la République qui déclara, devant le Congrès réuni à Versailles, le 22 juin 2009, que « la burqa n'est pas la bienvenue sur le territoire de la République française ». Le lendemain, la Conférence des présidents de l'Assemblée nationale décidait de créer une mission d'information «sur la pratique du port du voile intégral sur le territoire national » dont le rapport, remis le 26 janvier 2010

1. France. Conseil d'État, Étude relative aux possibilités juridiques d'interdiction du port du voile intégral, 30 mars 2010.

2. Cons. const., déc. 7 oct. 2010, n 2010-613 DC, Loi interdisant la dissimulation du visage dans l'espace public.

3. Loi $\mathrm{n}^{\circ}$ 2010-1192 du 11 octobre 2010 interdisant la dissimulation du visage dans l'espace public. 
au président de l'Assemblée ${ }^{4}$, concluait, unanimement, à l'incompatibilité de cette pratique avec les valeurs fondamentales de la République et préconisait l'adoption d'une résolution. Toutefois, ses membres n'étaient pas parvenus à s'accorder sur l'opportunité - et le dispositif - d'une loi d'interdiction et le président de la mission d'information lui-même en convenait : « Le débat national commen[çait] $»^{5}$ !

En deuxième lieu, parce que la mission d'information n'était pas parvenue à trancher, le groupe UMP déposait, le 5 février 2010, une proposition de loi «visant à interdire le port de tenues ou d'accessoires ayant pour effet de dissimuler le visage dans les lieux ouverts au public et sur la voie publique $»^{6}$.

En troisième lieu, le 11 mai 2010, l'Assemblée nationale, faisant usage du droit que le nouvel article 34-1 de la Constitution lui conférait, adoptait à l'unanimité une résolution «sur l'attachement au respect des valeurs républicaines face au développement de pratiques radicales qui y portent atteinte » par laquelle, notamment, les députés «considér[aient] que les pratiques radicales attentatoires à la dignité et à l'égalité entre les hommes et les femmes, parmi lesquelles le port d'un voile intégral, sont contraires aux valeurs de la République $»^{7}$.

C'est donc dans le cadre d'un débat à un double titre inédit que s'inscrivent le rapport du Conseil d'État et la décision du Conseil constitutionnel. Inédit, d'une part, par sa densité puisque quinze mois, seulement, séparent le lancement de l'idée à la tribune du Congrès de la publication de la loi au Journal officiel et que, pendant ces quinze mois, se succèdent un rapport parlementaire, une proposition de loi, un rapport du Conseil d'État, une résolution parlementaire, un débat législatif et une décision du Conseil constitutionnel. Inédit, d'autre part, par son souci de juridicité : à chacune des étapes que l'on vient de mentionner, sans cesse, fut posée la question de la constitutionnalité et de la conventionnalité du dispositif envisagé.

4. Rapport d’information sur la pratique du port du voile intégral sur le territoire national, Paris, Assemblée nationale, 26 janv. 2010, nº 2262.

5. Ibid. p. 13.

6. Proposition de loi visant à interdire le port de tenues ou d'accessoires ayant pour effet de dissimuler le visage dans les lieux ouverts au public et sur la voie publique, $\mathrm{n}^{\circ} 2283$, enregistrée à la présidence de l'Assemblée nationale le 5 févr. 2010.

7. Résolution sur l'attachement au respect des valeurs républicaines face au développement de pratiques radicales qui y portent atteinte, adoptée par l'Assemblée nationale le 11 mai 2010, TA n ${ }^{\circ} 459$. 
Ce rappel chronologique achevé, on en vient aux circonstances, elles aussi atypiques, dans lesquelles le Conseil d'État et le Conseil constitutionnel furent sollicités.

En premier lieu, c'est le 29 janvier 2010, soit trois jours après que la mission parlementaire d'information avait rendu un rapport d'une ampleur sans précédent ${ }^{8}$, que le Premier ministre appelait le Conseil d'État à jouer son rôle de conseiller juridique du Gouvernement, en vue d'« apporte[r] son concours au Gouvernement pour lui permettre d'arbitrer la traduction juridique des préoccupations exprimées par la représentation nationale et de soumettre rapidement au Parlement un projet de loi sur ce sujet». Il lui demandait spécialement d'étudier, d'ici la fin du mois de mars, "les solutions juridiques permettant de parvenir à une interdiction du port du voile intégral, qu['il souhaitait] la plus large et la plus effective possible».

En second lieu, le fait que la loi votée fût soumise au Conseil constitutionnel pourrait, de prime abord, ne rien avoir pour étonner. On aurait toutefois tort d'oublier que, en d'autres temps - et spécialement avant que n'existe la question prioritaire de constitutionnalité - le consensus républicain avait conduit à ce que des textes tout aussi âprement disputés échappassent à la saisine, telle la loi de 2004 sur le «voile à l'école »9 .

À cet égard encore, l'originalité de la loi de 2010 est manifeste. Parce que, telle une épée de Damoclès, l'hypothèque de l'inconstitutionnalité - et/ou de l'inconventionnalité - d'une loi d'interdiction générale pesait, la saisine a priori du Conseil constitutionnel fut très tôt envisagée. Plus encore et, contre toute attente, c'est Jean-François Copé, président du groupe majoritaire à l'Assemblée qui, très favorable à l'adoption d'un texte qui reprenait largement la proposition de loi qu'il avait déposée, le premier, en formula l'idée : «Pour qu'il n'y ait aucune ambiguité et parce que certains ont formulé des doutes que [le] vote, aussi large fût-il, ne saurait suffire à lever », il souhaitait

8. Pour mémoire, le rapport comptait 658 pages réunissant, outre un corpus de 170 pages et quinze propositions, les contributions de douze formations politiques représentées dans les deux chambres et de deux groupes politiques de l'Assemblée - l'une prenant la forme de propositions de résolution et de loi - et les contributions individuelles de douze députés membres de la mission. Rappelons en outre que la mission était composée de trente-deux députés issus des quatre groupes politiques représentés à l'Assemblée et mena six mois de travaux pendant lesquels deux cent onze personnes furent auditionnées, quatre déplacements en régions et à l'étranger organisés et des questionnaires adressés aux ambassades de France dans plus de trente États.

9. Loi n ${ }^{\circ} 2004-228$ du 15 mars 2004 encadrant, en application du principe de laïcité, le port de signes ou de tenues manifestant une appartenance religieuse dans les écoles, collèges et lycées publics. 
«que la loi votée soit soumise au Conseil constitutionnel avant sa promulgation, de sorte que son application ne puisse pas être contestée ${ }^{10}$. Le jour même, le président de l'Assemblée faisait savoir qu'il souscrivait à l'idée. Le 14 septembre, s'exprimant au Sénat au cours de la séance publique, Robert Badinter la reprenait, déclarant que «quel que soit le texte voté, il fa[llait] que le président du Sénat, comme celui de l'Assemblée nationale, le défère au Conseil constitutionnel ${ }^{11}$, si bien que lorsque, le jour même, la loi fut définitivement votée, c'est par une saisine identique que les présidents des deux assemblées - fait unique sous la V $\mathrm{V}^{\mathrm{e}}$ République - saisirent le Conseil constitutionnel sans qu'aucun moyen fût spécialement invoqué.

Ce n'est pas un mais deux prismes qu'il faudrait emprunter, puisque le Conseil d'État fut sollicité sur la faisabilité d'une loi qui, une fois votée contre son avis, fut, moyennant une réserve d'interprétation, déclarée conforme à la Constitution par le Conseil constitutionnel. Deux prismes donc, car deux postures, mais aussi, apparemment, parce que deux positions au fond.

Pour le Conseil d'État, dont le rapport est bref (35 pages), la situation peut être ainsi résumée : il déconseillait au Gouvernement de s'engager dans la voie d'une "interdiction générale et uniforme ${ }^{12}$ dont le fondement serait « juridiquement fragile ${ }^{13}$ et l'exposerait «à un très sérieux risque de censure ${ }^{14}$; il recommandait donc une solution jugée moins risquée : « harmoniser et, le cas échéant, renforcer le champ de l'interdiction de la dissimulation $\mathrm{du}$ visage, quelles que soient ses formes ${ }^{15}$. Il revenait au Gouvernement d'arbitrer et, quand bien même ce n'était peut-être pas sa première intention, le 21 avril, le Premier ministre indiquait qu'il était prêt à « prendre des risques juridiques» sur le sujet.

Brève, elle aussi (six considérants seulement), la décision du Conseil constitutionnel considère, par une formule sur laquelle on a, à juste titre, longuement glosé, que « Eu égard aux objectifs qu'il s'est assignés et compte tenu de la nature de la peine instituée [...], le législateur a adopté des dispositions qui assurent, entre la sauvegarde de l'ordre public et la garantie des droits constitutionnellement protégés, une conciliation qui n'est pas

10. JOAN CR 8 juill. 2010 , p. 5415.

11. JO Sénat CR 15 sept. 2010, p. 6760.

12. France. Conseil d'État, op. cit., p. 35.

13. Ibid. p. 26.

14. Ibid. p. 29.

15. Ibid. p. 37. 
manifestement disproportionnée ${ }^{16}$. Il ajoute une réserve, dans le même considérant, indiquant que « toutefois, l'interdiction de dissimuler son visage dans l'espace public ne saurait, sans porter une atteinte excessive à l'article 10 de la Déclaration de 1789, restreindre l'exercice de la liberté religieuse dans les lieux de culte ouverts au public $»^{17}$.

Toutefois, on aurait tort de s'en tenir à l'apparente contradiction des positions. Dans le cadre de l'office qui est le sien, c'est bien un choix politique que le Conseil constitutionnel valide, s'en tenant à un contrôle minimum, qui confirme, implicitement et sans surprise, que la Constitution ne lui « confère pas [...] un pouvoir général d'appréciation et de décision identique à celui du Parlement, mais lui donne seulement compétence pour se prononcer sur la conformité à la Constitution des lois déférées à son examen ${ }^{18}$ et que ce pouvoir d'appréciation est tributaire de ce que, paraphrasant la loi organique relative à l'application de l'article 61-1, on pourrait appeler un changement de circonstances, que le Conseil évoque lorsqu'il estime que « la loi déférée [a] pour objet de répondre à l'apparition de pratiques, jusqu'alors exceptionnelles, consistant à dissimuler son visage dans l'espace public ${ }^{19}$. Et parce que tout choix politique comporte des risques, il n'y a guère lieu de s'étonner que le Conseil d'État ait pris le parti, dans une perspective de conseiller juridique, d'attirer sur eux l'attention du Gouvernement.

Alors, si l'on admet que la contradiction résulte essentiellement de la posture, peut-être est-il possible de s'en tenir effectivement à un prisme unique. Réduisant l'apparente dualité à l'unicité, on admettrait que, de la lecture combinée de l'étude du Conseil d'État et de la décision du Conseil constitutionnel, ressortent deux idées partagées - que dans une certaine mesure l'arrêt de la Cour européenne des droits de l'homme ${ }^{20}$ viendra confirmer : en premier lieu, l'inadéquation à l'objectif poursuivi des fondements juridiques traditionnels (1) et, en second lieu, la nécessité d'un fondement novateur (2).

Disons-le une fois pour toutes, c'est la perspective de l'interdiction générale qui est ici explorée - quand bien même le Conseil d'État aurait mis en évidence qu'elle était risquée - et uniquement dans le cas de la dissimulation volontaire du visage puisqu'il est acquis - et le Conseil constitutionnel l'a

16. Cons. const., déc. 7 oct. 2010 , précit., consid. 5 .

17. Ibid.

18. La formule est constamment reprise depuis la décision n 74-54 DC du 15 janvier 1975 dite «IVG» (cons. 1).

19. Cons. const., déc. 7 oct. 2010, précit., consid. 4.

20. CEDH, Gde ch., $1^{\text {er }}$ juill. 2014, n 43835/11, S.A.S. c/ France. 
confirmé sans même le motiver - que ne peut qu'être interdite la dissimulation forcée $e^{21}$.

\section{L'INADÉQUATION DES FONDEMENTS JURIDIQUES « CLASSIQUES ॥}

Si le Conseil d'État est, compte tenu de sa posture, plus explicite sur le sujet, nul doute que le Conseil constitutionnel partage avec lui la conviction qu'il est, dans la perspective d'une interdiction générale de la dissimulation du visage, des fondements juridiques impossibles (1.1) et un fondement juridique risqué (1.2).

\subsection{LES FONDEMENTS IMPOSSIBLES}

Proche sur ce point des conclusions du rapport de la mission parlementaire d'information, le Conseil d'État expliquait, de manière détaillée, les raisons pour lesquelles le principe de laïcité, les principes fondamentaux de protection de la dignité et de l'égalité entre les hommes et les femmes et la protection de la sécurité publique étaient, isolément, insusceptibles de fonder une interdiction générale du port du voile intégral. Le législateur l'ayant sur ce point partiellement entendu, le laconisme du Conseil constitutionnel atteste de leur convergence de vue.

En premier lieu, le principe de laïcité, bien que constitutionnellement fondé, n'impose que «la stricte neutralité de l'État et des collectivités visà-vis des pratiques religieuses, et réciproquement ${ }^{22}$. Il ne saurait dès lors, hors de ce cadre, emporter d'obligations à la charge des particuliers « qu'en raison d'exigences propres à certains services publics (comme c'est le cas des établissements scolaires) ${ }^{23}$. D'où le Conseil d'État déduisait, sans surprise, que «le principe de laïcité ne pourrait donc, à lui seul, fonder une interdiction générale du port du voile intégral ${ }^{24}$.

21. Le considérant 6 de la décision $n^{\circ}$ 2010-613 DC lui est consacré qui considère "que l'article 4 de la loi déférée, qui punit d'un an d'emprisonnement et de $30000 €$ d'amende le fait d'imposer à autrui de dissimuler son visage " n'est pas contraire à la Constitution; sans même l'évoquer, l'étude du Conseil d'État était consacrée au port volontaire du voile intégral.

22. France. Conseil d'État, op. cit., p. 18.

23. Ibid.

24. Ibid. 
On ajoutera que l'application du principe de laïcité eut en outre supposé que le port du voile intégral fût seul visé - ce qui correspond à la demande que le Premier ministre avait adressée au Conseil d'État - et que celui-ci fût analysé comme la manifestation d'une conviction religieuse. C'est sans doute en écho à cet aspect du débat que le Conseil constitutionnel fit le choix de formuler une réserve d'interprétation concernant les lieux de culte, manifestant en outre ainsi qu'il était attentif à la jurisprudence de la Cour européenne des droits de l'homme et, spécialement, à un arrêt du 23 février 2010 Ahmet Arslan c/ Turquie ${ }^{25}$, d'ailleurs reproduit au dossier documentaire du Conseil, et qui fut présenté lors de sa publication comme faisant obstacle à ce que le législateur français pût adopter la loi sur laquelle le débat était depuis plusieurs mois engagé.

En deuxième lieu, c'est «l'invocation des principes fondamentaux de protection de la dignité et de l'égalité entre les hommes et les femmes, isolément ou combinés ${ }^{26}$ qui est écartée par le Conseil d'État, au motif que lesdits principes ne peuvent faire obstacle à l'exigence de protection du libre arbitre et, dès lors, «s'appliquer à des personnes qui ont choisi délibérément le port du voile intégral ${ }^{27}$. On ajoutera, ainsi que le Conseil d'État le suggère, qu'invoquer isolément ou en combinaison ces deux fondements, reviendrait à porter un jugement de valeur sur la pratique du port du voile, ce qui, assurément, n'est pas le rôle du droit.

Le principe de dignité n'est pas même évoqué par le Conseil constitutionnel. Il intervient, en revanche, sur le terrain du principe d'égalité entre les hommes et les femmes puisque, outre qu'il cite expressément le troisième alinéa du préambule de la Constitution de $1946^{28}$, le Conseil reprend en quelque sorte à son compte le jugement de valeur porté par le législateur dans le cas du voile intégral, rappelant que ce dernier « a estimé que les femmes dissimulant leur visage, volontairement ou non, se trouvent placées dans une situation d'exclusion et d'infériorité manifestement incompatible avec les principes constitutionnels de liberté et d'égalité ${ }^{29}$. Rejoignant partiellement le Conseil d'État, c'est donc égalité et liberté qui se trouvent ainsi combinées.

En troisième lieu, le Conseil d'État écarte, en des termes lapidaires, la possible invocation de la sécurité publique, estimant à juste titre que «le

25. CEDH, 23 févr. 2010, n 41135/98, Ahmet Arslan et autres c/ Turquie.

26. France. Conseil d'État, op. cit., p. 19.

27. Ibid., p. 20.

28. Cons. const., déc. 7 oct. 2010, précit., consid. 3.

29. Ibid., consid. 4. 
voile intégral n'a pas en effet, en tant que tel et à ce jour, soulevé de problèmes de sécurité publique particuliers, de troubles à l'ordre public ou de réactions violentes de nature à justifier une interdiction générale de son port pour ce motif $»^{30}$. On entend le contre-argument qui consisterait à dire que mieux vaut prévenir que guérir et que l'on aura beau jeu, si le pire devait survenir, de dire que le droit faisait obstacle à une interdiction désormais devenue nécessité. La position du Conseil d'État n'en demeure pas moins, sur ce point, incontestable et l'on se plaît à penser que le constat sera durable.

Une fois encore, le Conseil constitutionnel semble légèrement diverger, lorsque, citant les justifications du législateur, il rappelle que « de telles pratiques peuvent constituer un danger pour la sécurité publique ${ }^{31}$. Outre que lui est immédiatement adjointe la méconnaissance des « exigences minimales de la vie en société », le Conseil constitutionnel tempère ce fondement en indiquant que par « les dispositions déférées, le législateur a ainsi complété et généralisé des règles jusque-là réservées à des situations ponctuelles à des fins de protection de l'ordre public ${ }^{32}$. Comme l'on sait que le Conseil constitutionnel a une conception ancienne et stricte de l'ordre public articulée autour du triptyque "sécurité, tranquillité, salubrité ", on en déduira que, comme le Conseil d'État, il considère que dans sa composante sécurité il ne peut fonder que des limitations ponctuelles.

À juste titre, le Conseil d'État ajoutait qu'une interdiction du port du seul voile intégral poserait difficulté, raison pour laquelle la question doit être examinée «sous l'angle, plus large, de la dissimulation du visage ${ }^{33}$. Lorsque le Conseil constitutionnel se prononce, la question est évidemment tranchée. Si l'affirmation relève, en droit, de l'évidence, les arguments invoqués dans le rapport peuvent surprendre puisque, juridiquement, le droit de l'Union européenne est principalement visé, ce qui pourrait laisser penser que la conception française du principe d'égalité pourrait s'en accommoder.

Rien d'étonnant donc, ni même de novateur, dans le constat du Conseil d'État, particulièrement si l'on songe que la mission parlementaire d'information était parvenue peu ou prou aux mêmes conclusions : le risque de censure d'une loi d'interdiction générale invoquant les fondements auxquels on a le plus spontanément pensé est avéré, raison pour laquelle il doit y être renoncé. En revanche, c'est un risque juridique présumé qui

30. France. Conseil d’État, op. cit., p. 20.

31. Cons. const., déc. 7 oct. 2010, précit., consid. 4 .

32. Ibid.

33. France. Conseil d'État, op. cit., p. 21. 
conduit le Conseil d'État à écarter un fondement possible au motif qu'il est « inédit».

\subsection{UN FONDEMENT « INÉDIT » RISQUÉ}

Le Conseil d'État consacre des développements substantiels au « seul fondement possible ${ }^{34}$ d'une interdiction générale : l'ordre public. À l'aune du Conseil d'État, le raisonnement dépasse le triptyque précédemment évoqué puisque l'on sait qu'il y a intégré, à différentes époques, une quatrième composante et a, par conséquent, admis que la notion pût être «flottante ». Il évoque, en particulier, l'idée d'un « ordre public non matériel ${ }^{35}$ qui, parce qu'elle reposerait sur une « conception renouvelée de l'ordre public, étrangère à la jurisprudence actuelle du Conseil constitutionnel et donc juridiquement fragile ${ }^{36}$, ne saurait être recommandée comme fondement d'une interdiction générale, "sous peine de soumettre une prohibition générale [...] à un très sérieux risque de censure $»^{37}$.

Le raisonnement n'est assurément pas erroné et c'est par précaution - voire par précautionnisme - juridique que le Conseil d'État renonce à cette piste, arguant au surplus d'obstacles tenant à la nécessité de prévoir « des exceptions nombreuses et délicates à définir ${ }^{38}$ et recommandant plutôt que les parlementaires s'en tiennent à une résolution qui «mettrait en valeur les éléments constitutifs du contrat social tels qu'ils apparaîtraient à la représentation nationale $»^{39}$.

Disons-le, pour deux raisons au moins, l'argument n'apparaissait d'emblée pas convaincant. D'une part, parce que le risque de censure, s'il était sérieux, ne pouvait à lui seul justifier de renoncer à un choix de nature politique. De manière plus générale, le risque juridique, invoqué comme une antienne tout au long du rapport, est le lot commun de tout dispositif législatif nouveau palliant un vide juridique, a fortiori, s'il consiste en une interdiction générale. L'état du droit ne pouvait donc être en soi un obstacle, sauf à faire le choix de principe de l'immobilisme juridique et de l'immutabilité des règles de droit. D'autre part, parce qu'il n'était pas assuré que la jurisprudence

34. France. Conseil d’État, op. cit., p. 24.

35. Ibid., p. 25.

36. Ibid., p. 26.

37. Ibid., p. 29.

38. Ibid., p. 28.

39. Ibid., p. 28. 
constitutionnelle et européenne fût, isolément ou de manière combinée, d'une cohérence si parfaite que l'argument d'un ordre public non matériel dût être d'emblée rejeté. On pouvait déjà, à l'époque, penser, par exemple, aux jurisprudences relatives au regroupement familial ou au PACS, que le Conseil d'État mentionnait d'ailleurs, mais en en tirant des conclusions qui pouvaient être renversées ${ }^{40}$. Dans l'un et l'autre cas, c'est bien au nom de l'ordre public que sont respectivement rappelées les limites que constituent, pour le regroupement familial, la polygamie et, pour le PACS, la prohibition de l'inceste. Sans qu'il soit possible, non plus que nécessaire, d'identifier une composante de l'ordre public à laquelle ces deux justifications pourraient être rattachées, nul ne contestera que ne sont en cause ni la sécurité, ni la tranquillité, ni la salubrité publiques et pas davantage la moralité ou la dignité ! L'argument du Conseil d'État selon lequel « les pratiques en cause ne sont pas de même nature que la dissimulation du visage dans l'espace public, dès lors qu'elles touchent aux relations intimes entre personnes, et par là, à l'ordre même de la société ${ }^{41}$ pouvait être discuté, car si l'on admet que le port du voile relève du libre arbitre et de la liberté personnelle, comment nier qu'il s'apparente à l'intime et que, hors du domicile privé, il met en cause les relations entre personnes...

On arguera, bien sûr, que le Conseil d'État était dans son rôle et que la rigueur juridique imposait qu'il mît en garde le Gouvernement contre le risque juridique excessif inhérent à une conception de l'ordre public périlleuse parce qu'entièrement renouvelée. Sans nier la pertinence du raisonnement, on peut s'étonner que la piste ait été écartée aussitôt qu'esquissée au seul motif qu'elle reposait sur une " définition inédite ${ }^{42}$ ou une « conception inédite ${ }^{43}$ de l'ordre public, a fortiori compte tenu de la complexité du dispositif que le Conseil lui préférait in fine. Point n'est ici besoin de longuement s'y attarder puisqu'elle n'a pas finalement été retenue, c'est une interdiction partielle que le Conseil d'État recommandait parce qu'exempte de risques juridiques. Selon lui, la solution la moins risquée consiste à « harmoniser et, le cas échéant, renforcer le champ de l'interdiction de la dissimulation du visage, quelles que soient ses formes ${ }^{44}$. C'est, en poussant la logique à l'extrême, ce que le législateur a fait et le Conseil constitutionnel le reconnaît.

40. Ibid., p. 27.

41. Ibid.

42. Ibid., p. 26.

43. Ibid., p. 27.

44. Ibid., p. 37. 
La solution préconisée par le Conseil d'État était minimale et visait à corriger l'hétérogénéité du droit positif que la présentation liminaire de son état avait confirmée. Il précisait d'ailleurs que «si une telle démarche était retenue, il conviendrait cependant, au regard des exigences précédemment rappelées, que le cumul de ces interdictions ne confine pas à une interdiction générale, ni par l'effet des dispositions législatives ou réglementaires qui pourraient être adoptées, ni du fait de l'exercice des pouvoirs de police des autorités compétentes ${ }^{45}$. Il confirmait ainsi, indiscutablement, le rejet d'un ordre public non matériel et de son esprit même, puisque sa préconisation s'inscrivait dans une démarche quantifiable et de proportionnalité - voire proportionnaliste - qui permettait que chaque interdiction puisse être précisément appréciée et pesée. Fort logiquement, les deux fondements proposés étaient alternatifs et classiques : sauvegarde de la sécurité publique et obligation, par nature ponctuelle, "procéd[ant] de la nécessaire reconnaissance de la personne dans certains lieux " liée aux exigences spécifiques de bon fonctionnement de certains services publics ou de l'application de dispositions qui comportent des restrictions ou des distinctions liées à l'identité ou à l'âge. L'un et l'autre ne pouvaient conduire qu'à une interdiction dont l'application spatiale et temporelle devait être limitée.

Dit autrement, à raison de sa posture, le Conseil d'État recommandait le fondement le plus classique car le plus assuré, tout en reconnaissant qu'il ne permettait pas d'atteindre l'objectif que le Gouvernement se fixait. Symétriquement, il esquissait un fondement novateur qu'il récusait immédiatement à raison de sa prétendue fragilité. C'est donc un fondement novateur peu éloigné de celui que le Conseil d'État envisageait qui fut retenu par le législateur et dont le Conseil constitutionnel confirma la constitutionnalité.

\section{LA NÉCESSITÉ D'UN FONDEMENT NOVATEUR}

Reconnaissons-le d'emblée, le Conseil constitutionnel autant que le législateur ont peiné à très exactement cerner le fondement de l'interdiction générale de la dissimulation du visage (2.1) ; donnant en cela partiellement raison au Conseil d'État, le juge constitutionnel a néanmoins mis en lumière une norme de référence jusque-là inexploitée (2.2).

45. Ibid. 


\subsection{LA « JUSTE ॥ CONCILIATION}

C'est en définitive l'équilibre trouvé par le législateur qui conduit le Conseil constitutionnel à considérer que l'interdiction générale de la dissimulation du visage est juridiquement fondée. «Eu égard aux objectifs qu'il s'est assignés et compte tenu de la nature de la peine instituée [...], le législateur a adopté des dispositions qui assurent, entre la sauvegarde de l'ordre public et la garantie des droits constitutionnellement protégés, une conciliation qui n'est pas manifestement disproportionnée ${ }^{46}$ : tel est le motif principal au soutien de la déclaration de conformité. Apparemment clair autant que classique, il procède, ainsi que le confirme le commentaire autorisé de la décision aux Cahiers du Conseil constitutionnel, à une balance des intérêts constitutionnels en présence. Toutefois, on ne saurait se limiter à ce constat et, à trois égards au moins, le raisonnement mérite d'être plus amplement analysé.

Tout d'abord, bien que de proportionnalité, le contrôle auquel le Conseil a procédé a toutes les allures d'un contrôle minimum. C'est, en effet, l'absence de disproportion manifeste de la conciliation qui est affirmée, compte tenu des impératifs entre lesquels le législateur devait arbitrer. On l'a déjà dit : la Constitution ne lui « confère pas [...] un pouvoir général d'appréciation et de décision identique à celui du Parlement, mais lui donne seulement compétence pour se prononcer sur la conformité à la Constitution des lois déférées à son examen ${ }^{47}$.

Le raisonnement est, ensuite, conforté par les normes constitutionnelles auxquelles le Conseil a choisi de se référer ${ }^{48}$ qui, toutes ont en commun, de fonder la compétence du législateur pour encadrer l'exercice des libertés. Ainsi en est-il, bien sûr et au premier chef, de l'article 4 de la Déclaration des droits de l'homme et du citoyen de 1789 qui reconnaît au seul législateur compétence pour déterminer les bornes mises à « l'exercice des droits naturels de chaque homme [...] qui assurent aux autres membres de la Société la jouissance de ces mêmes droits ». Il en va de même, assurément, quoique dans un cadre plus circonscrit, de l'article 10 de la Déclaration et de l'alinéa 3 du Préambule de 1946 qui affirment, respectivement, les limites qui peuvent être apportées à la liberté d'opinion afin que sa « manifestation ne trouble pas

46. Cons. const., déc. 7 oct. 2010, précit., consid. 5 .

47. Cons. const., déc. 15 janv. 1975, n 74-54 DC, Loi relative à l'interruption volontaire de la grossesse.

48. Cons. const., déc. 7 oct. 2010, précit., consid. 3 . 
l'ordre public établi par la loi » (ordre public dont le périmètre pourrait aller au-delà du triptyque) et la garantie de l'égalité hommes-femmes qu'il incombe à la loi d'assurer. Mais il en va de même, enfin et surtout, de l'article 5 de la Déclaration qui, en énonçant par une formule évidemment restrictive que le législateur n'a le droit de défendre que "les actions nuisibles à la Société », n'en constitue pas moins une norme d'habilitation aux termes de laquelle le législateur est seul à même d'apprécier ce que sont lesdites actions et, pour ce seul motif, de les défendre, c'est-à-dire les interdire. Que le Conseil ait ici et pour la première fois, de son propre chef, choisi de se référer à cette disposition n'est à l'évidence pas anodin ; a fortiori si on le met en relation avec l'examen minutieux de l'intention du législateur auquel il procède.

Car, enfin, ce sont cette intention et, tout spécialement, les « objectifs que [le législateur] s'est assignés ${ }^{49}$, qui justifient que le dispositif soit validé. En témoigne le considérant 4 de la décision par lequel le Conseil développe longuement les circonstances qui ont incité le législateur, compte tenu de l'évolution de certaines pratiques, à " complét[er] et généralis[er] des règles jusque-là réservées à des situations ponctuelles à des fins de protection de l'ordre public ».

Il n'est pas insignifiant que la nature de la peine instituée soit aussi présentée comme participant de ce juste équilibre, que le Conseil en quelque sorte précise par la réserve d'interprétation qu'il formule à l'égard des lieux de culte. Fût-elle fort classique, il n'était pas avéré que le Conseil constitutionnel jugeât nécessaire de la formuler. Il le fait pourtant dans la foulée, considérant que « l'interdiction de dissimuler son visage dans l'espace public ne saurait, sans porter une atteinte excessive à l'article 10 de la Déclaration de 1789, restreindre l'exercice de la liberté religieuse dans les lieux de culte ouverts au public ${ }^{50}$. Il précise ainsi les termes de la conciliation sans qu'il soit, par ailleurs, avéré que ce fût une nécessité. En effet, s'il ne fait aucun doute que les lieux de culte sont des lieux ouverts au public au sens de l'article 2 de la loi et que, par conséquent, l'interdiction de la dissimulation du visage dût y être appliquée, il n'est pas certain qu'il y ait restriction à la liberté religieuse puisqu'aucune religion n'impose la dissimulation du visage ; dans le cas particulier du voile intégral, les représentants de la communauté musulmane l'ont à plusieurs reprises rappelé. Toutefois, le port volontaire du voile intégral étant souvent motivé ou inspiré par une conviction religieuse, le choix du Conseil constitutionnel peut sembler justifié, spécialement au

49. Ibid, consid. 5 .

50. Ibid. 
regard de la jurisprudence de la Cour européenne des droits de l'homme qui, au vu du dossier documentaire du Conseil, a, sur ce point, été examinée. Nul véritable étonnement donc, à ce que le Conseil ait jugé utile de préciser ainsi les termes de la conciliation que le législateur a entendu opérer et même, disons-le, une manière de confirmation de ce que le contrôle de constitutionnalité, eût-il les apparences d'un contrôle minimum, est bien de proportionnalité.

Il n'y aurait, en définitive, rien de très novateur dans la démarche du législateur non plus que dans la décision du Conseil. Pourtant, il semble bien que ce soit une nouvelle norme de référence que le Conseil a dégagée, fût-ce de manière dissimulée.

\subsection{UNE NORME DE RÉFÉRENCE « DISSIMULÉE 》}

On déduirait un peu rapidement du considérant central de la décision que c'est la sauvegarde de l'ordre public qui justifie l'interdiction générale nouvelle que le législateur a posée. La référence à l'article 5 de la Déclaration de 1789 est à cet égard essentielle qui, sans conduire à l'identification d'une nouvelle composante de l'ordre public, incite à une réflexion sur le caractère mouvant et extensif de l'intérêt général et ce que le Conseil d'État considérait comme un écho au contrat social. Car, in fine, c'est la vie en société qui, plus sûrement que tout autre, est le fondement de la loi et l'article 5 de la Déclaration confirme que le législateur est habilité à en déterminer sinon le contenu, du moins les contours.

Dit autrement, il revient au seul législateur d'apprécier l'opportunité d'adopter de nouveaux dispositifs adaptés aux mutations de la société ; plus complexe se fait évidemment le raisonnement, mais assurément, ce sont d'abord "les exigences minimales de la vie en société » que le législateur a entendu préserver ${ }^{51}$. Ici réside, sans doute, le point le plus novateur de la décision du Conseil, en même temps, disons-le, que le plus embarrassé, puisqu'il semble résulter de la lecture combinée de ses considérants 4 et 5 que l'objectif de valeur constitutionnelle de sauvegarde de l'ordre public que le législateur a, à bon droit et de manière proportionnée, poursuivi recouvre tout à la fois la «sécurité publique », les « exigences minimales de la vie en société » et, peut-être même, l'élimination de "situation[s] d'exclusion et d'infériorité manifestement incompatible[s] avec les principes constitutionnels

51. Ibid., consid. 4. 
de liberté et d'égalité ». Pour autant, le pas n'est pas clairement franchi qui consisterait à reconnaître l'existence d'un ordre public immatériel, manière sans doute pour le Conseil de préserver l'avenir et ne pas ouvrir la boîte de Pandore. En revanche, de cette combinaison résulte peut-être une lecture renouvelée de l'intérêt général ou de l'intérêt de la société puisque le législateur, par le biais de l'article 5, serait habilité à prohiber tout comportement qui pourrait s'analyser comme de nature à lui nuire.

La logique de la Déclaration est clairement préservée, puisque nombreux sont ces termes qui pourraient être invoqués au soutien d'un ordre public immatériel ou d'un intérêt général en constante redéfinition, si l'on admet que c'est bien un ordre social que ses auteurs avaient à l'esprit lorsqu'ils tentaient d'établir le cadre des rapports entre homme et citoyen, d'une part, et société constituée, d'autre part. Il n'est à cet égard pas anodin que la jurisprudence européenne accorde une place importante à la marge d'appréciation des États ainsi qu'aux exigences d'une société démocratique, allant jusqu'à considérer que «le pluralisme et la démocratie doivent également se fonder sur le dialogue et un esprit de compromis, qui impliquent nécessairement de la part des individus des concessions diverses qui se justifient aux fins de la sauvegarde et de la promotion des idéaux et valeurs d'une société démocratique ${ }^{52}$. L'argument, couramment présenté au titre des "spécificités du contrôle de la Cour européenne des droits de l'homme », selon lequel celle-ci recourt à des "éléments de contexte étrangers à l'office du juge national », telle "l'existence éventuelle d'un "consensus européen" sur la question qui lui est posée " ${ }^{53}$, doit à l'évidence et pour deux raisons être nuancé : en premier lieu, parce qu'il n'est pas conforme à l'idée même de marge nationale d'appréciation qui postule que chaque État puisse, pour des motifs légitimes, faire valoir une spécificité, et, en second lieu, parce que les débats et controverses dans des États voisins laissent penser que la France n'est d'ores et déjà pas isolée. C'est d'ailleurs au nom d'un «vivre ensemble » que l'on peut considérer comme «à la française " que, in fine, la Cour européenne arguera de la marge d'appréciation pour considérer que l'interdiction de la dissimulation du visage n'emporte pas violation de la Convention.

En définitive, on le voit, c'est la posture bien davantage que le fond qui sépare Conseil d'État et Conseil constitutionnel. Pour l'un et l'autre, le prisme

52. CEDH, 4 déc. 2008, n³1645/04, Kervanci c/ France et n² 27058/05, Dogru c/ France, pt. 62.

53. France. Conseil d'État, op. cit., p. 32. 
est identique : une interdiction générale doit demeurer l'exception, justifiant que son fondement fasse l'objet d'une appréciation qui sorte elle-même un peu de l'ordinaire. Exigences minimales de la vie en société, ordre public immatériel ou intérêt général ne sont finalement que d'autres expressions pour dénommer ce que la Cour européenne appellera le «vivre ensemble ». Trop vague, dira-t-on, mais n'oublions pas que, en démocratie, le droit, aussi essentiel fût-il, ne saurait faire obstacle au politique! 